\title{
On the importance of low-energy beta beams for supernova neutrino physics
}

\author{
N. Jachowicz ${ }^{1, \mathrm{a}}$ and G.C. McLaughlin ${ }^{2, \mathrm{~b}}$ \\ 1 Department of Subatomic and Radiation Physics, Ghent University, Proeftuinstraat 86, B-9000 Gent, Belgium \\ 2 Department of Physics, North Carolina State University, Raleigh, NC 27695-8202, USA
}

Received: 17 June 2005 /

Published online: 21 February 2006 - (C) Società Italiana di Fisica / Springer-Verlag 2006

\begin{abstract}
We show that low-energy beta beams are very well suited to obtain information about supernovaneutrino interactions. Linear combinations of low-energy beta-beam spectra are fitted to supernovaneutrino energy distributions. The resulting synthetic spectra are able to reproduce the response of a nuclear target to an incoming supernova neutrino flux in a very accurate way. This can provide important information about the neutrino response in a terrestrial detector. We illustrate this technique using deuterium and ${ }^{16} \mathrm{O}$ as target material. The procedure provides an easy and straightforward way to apply the results of a beta-beam neutrino-nucleus measurement to a supernova neutrino detector, virtually eliminating potential uncertainties due to nuclear-structure calculations.
\end{abstract}

PACS. 25.30.Pt Neutrino scattering - 26.50.+x Nuclear physics aspects of novae, supernovae, and other explosive environments - 97.60.Bw Supernovae - 13.15.+g Neutrino interactions

\section{Introduction}

The supernova problem is a longstanding one. Since ancient times, literature has reported the observation of suddenly appearing bright new objects in the sky. While the name of the phenomenon reflects the confusion the origin of these events caused, today the general nature of core-collapse supernovae is understood. Notwithstanding the progress that has been made, the modeling of corecollapse supernova events and in particular the generation of successful explosions still seems to face major problems. The neutrinos from supernova 1987A confirmed that weak interactions play an important role in the explosion mechanism. Hence, neutrinos from the next galactic corecollapse supernova are much anticipated, since they are the only particles giving us the chance to see deep into the center of the event and shed light on the processes driving the explosion mechanism.

The terrestrial detection of supernova neutrinos can provide a broad variety of information $[1,2]$. The arrival times of the neutrinos are related to their mass and can moreover hint at the fate of the star [2,3]. Several reactions provide directional information, important for optical telescopes awaiting the photons in the wake of the supernova neutrino flux. The energy of the neutrinos can be inferred from the energy of the decay products. It indicates the de-

\footnotetext{
a e-mail: natalie.jachowicz@ugent.be

b e-mail: Gail_McLaughlin@ncsu.edu
}

coupling site of the neutrinos and the temperature there. As mu and tau supernova neutrinos do not have enough energy to produce a massive lepton in a charged-current reaction, the flavor of the arriving neutrinos can be inferred from the frequency differences between the occurrence of neutral and charge-exchange processes. Whether a neutrino or an antineutrino entered the detector can be determined by looking at the charge of the outgoing lepton for electron (anti)neutrinos or by examining the spin of the outgoing nucleon in neutral-current nucleon knockout off nuclei $[4,5]$. When the signal in the detector is accurately resolved, the observed neutrino energies and flavors can help to disentangle the mixing scheme induced by oscillations $[6,7,8]$.

Nuclei have relatively large cross-sections for neutrino reactions and are energy-sensitive in the range of interest, several particle-emission thresholds opening up with increasing incoming neutrino energies. This makes nuclear targets important as detecting material. Galactic supernova neutrinos could be detected by existing and proposed supernova neutrino detectors such as SNO [9], SuperKamiokande [10], KamLAND [1], LVD [11], MiniBooNe [12], OMNIS [13], LAND [14], and LENA [15]. Favored detection nuclei are ${ }^{12} \mathrm{C},{ }^{16} \mathrm{O},{ }^{56} \mathrm{Fe},{ }^{208} \mathrm{~Pb}$, and deuterium. However, the signal in the detector can only be interpreted as well as the relevant neutrino-nucleus cross-sections are understood. For most nuclei very little experimental neutrino data exists in the relevant energy 
Table 1. Percentage of the original ion flux that will be converted to neutrinos entering the target for the detector setup described in [22].

\begin{tabular}{c|c||c|c}
\hline$\gamma$ & $\%$ & $\gamma$ & $\%$ \\
\hline 2 & 0.1 & 10 & 8 \\
3 & 0.5 & 15 & 14 \\
5 & 2 & & \\
\hline
\end{tabular}

region. This is due to the very small cross-sections for weak-interaction processes, and an additional limitation is caused by the fact that monochromatic neutrino beams are not available $[16,17]$. This has as a consequence that for most applications one has to rely on theoretical predictions, with their related uncertainties and model dependencies [18].

Beta beams, which are neutrino beams produced by the beta decay of nuclei that have been accelerated to high gamma factor, were original proposed for high-energy applications, such as the measurement of the third neutrino mixing angle $\theta_{13}$ [19]. Volpe $[20,21,22]$ suggested that a beta beam run at lower gamma factor, would be useful for neutrino measurements in the tens of $\mathrm{MeV}$ range.

We suggest exploiting the flexibility these beta-beam facilities offer [23], combined with the fact that beta-beam neutrino energies overlap with supernova neutrino energies, to construct "synthetic" spectra that approximate an incoming supernova neutrino energy distribution. Using these constructed spectra we are able to reproduce total and differential folded supernova neutrino cross-sections very accurately.

\section{Synthetic spectra}

Traditionally, supernova neutrino energy distributions were parametrized using Fermi-Dirac distributions. The spectra are however not purely thermal, as the decoupling sites of the neutrinos are influenced by their flavor and energy, leading to the use of "effective temperatures" and "effective chemical potentials" in these distributions. Recent calculations showed that descriptions of a supernova neutrino spectrum are provided by a power law distribution [24]:

$$
n_{S N[\langle\varepsilon\rangle, \alpha]}(\varepsilon)=\left(\frac{\varepsilon}{\langle\varepsilon\rangle}\right)^{\alpha} e^{-(\alpha+1) \frac{\varepsilon}{\langle\varepsilon\rangle}},
$$

where $\langle\varepsilon\rangle$ and $\alpha$ represent the average energy and the width of the spectrum, respectively. The average neutrino energy $\langle\varepsilon\rangle$ is related to the temperature at the decoupling site, and the effect of $\alpha$ is equivalent to that of the introduction of the effective chemical potential in the Fermi-Dirac distribution. Neutrino-nucleus reaction crosssections depend on the square of the incoming energy, thus rising very fast with neutrino energies. Hence, the folded cross-sections reach their maximum at much higher energy values than the supernova neutrino energy spectrum does. Typically even neutrinos with energies more than twice
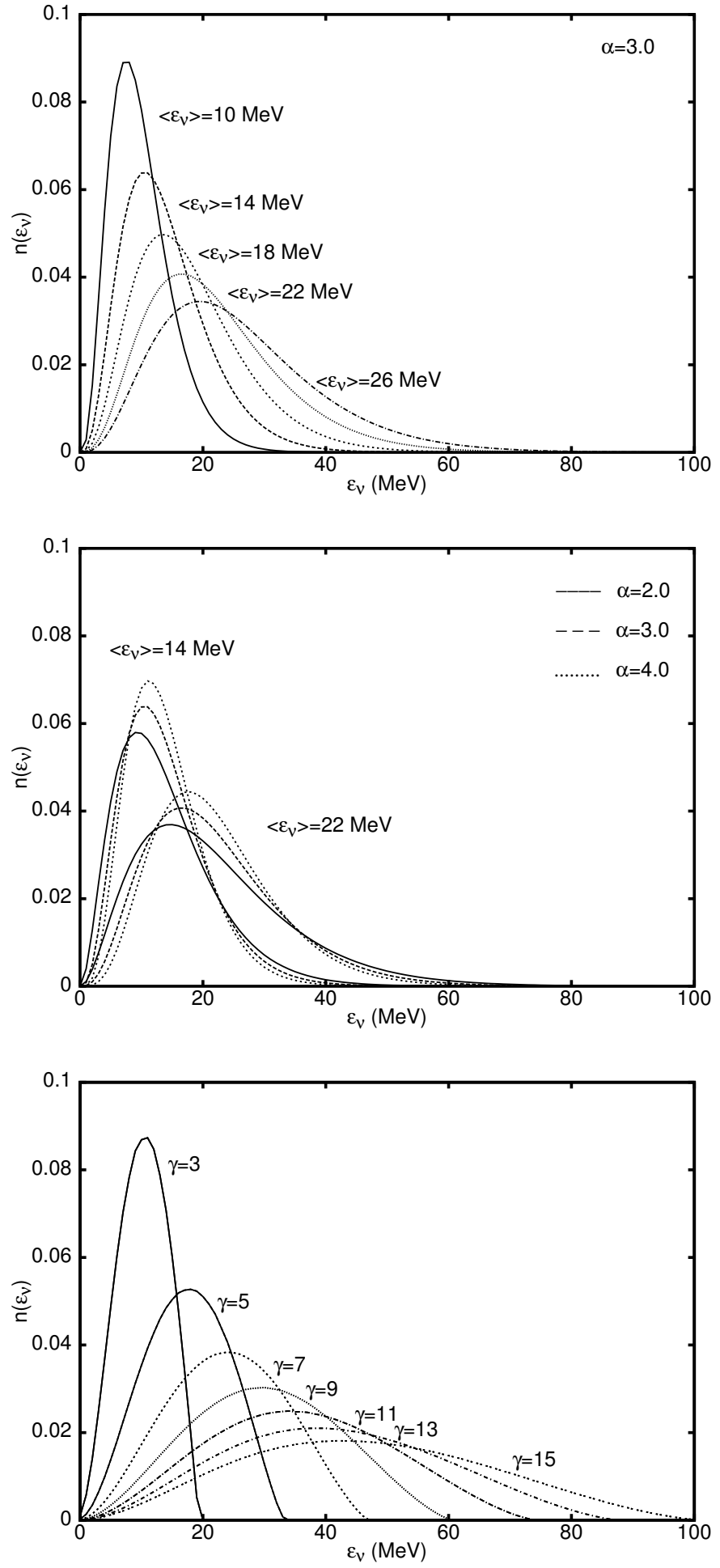

Fig. 1. Comparison between neutrino spectra. The top panel shows the power law parametrization for different values of the average neutrino energy. The width parameter is kept fixed at $\alpha=3$. The middle panel compares spectra with different widths for average energies of 14 and $22 \mathrm{MeV}$. The bottom panel shows beta-beam spectra stemming from ${ }^{18} \mathrm{Ne}$ decay for different values of the boost parameter $\gamma$. 
Table 2. Parameters for the best fit to the supernova neutrino spectrum, defined by average energy $\langle\varepsilon\rangle$ and width $\alpha$ as indicated in the first columns of the table. The left part of the table presents parameterizations $\left(a_{i=1,2,3}^{(3)}, \gamma_{i=1,2,3}^{(3)}\right)$ for synthetic spectra constructed with three normalized gamma spectra, the right part of the table shows the parametrization $\left(a_{i=1, \ldots, 5}^{(5)}, \gamma_{i=1, \ldots, 5}^{(5)}\right)$ for spectra obtained as the linear combination of five normalized gamma spectra stemming from ${ }^{18} \mathrm{Ne}$ decay.

\begin{tabular}{cc||cccccc|ccccccccccc}
\hline$\langle E\rangle$ & $\alpha$ & $a_{1}^{(3)}$ & $\gamma_{1}^{(3)}$ & $a_{2}^{(3)}$ & $\gamma_{2}^{(3)}$ & $a_{3}^{(3)}$ & $\gamma_{3}^{(3)}$ & $a_{1}^{(5)}$ & $\gamma_{1}^{(5)}$ & $a_{2}^{(5)}$ & $\gamma_{2}^{(5)}$ & $a_{3}^{(5)}$ & $\gamma_{3}^{(5)}$ & $a_{4}^{(5)}$ & $\gamma_{4}^{(5)}$ & $a_{5}^{(5)}$ & $\gamma_{5}^{(5)}$ \\
\hline 14 & 3 & 0.97 & 5 & 0.03 & 8 & & & 0.97 & 5 & 0.03 & 8 & & & & & \\
18 & 2 & 0.77 & 5 & 0.18 & 8 & 0.05 & 11 & 0.73 & 5 & 0.12 & 7 & 0.06 & 8 & 0.06 & 9 & 0.03 & 12 \\
18 & 4 & 0.86 & 5 & 0.05 & 7 & 0.09 & 8 & 0.86 & 5 & 0.07 & 7 & 0.05 & 8 & 0.01 & 9 & 0.01 & 10 \\
22 & 3 & 0.77 & 6 & 0.19 & 9 & 0.04 & 12 & 0.56 & 5 & 0.16 & 7 & 0.15 & 8 & 0.11 & 10 & 0.02 & 13 \\
\hline
\end{tabular}

the average energy of the distribution make sizable contributions to the folded cross-section, and integrated crosssections only converge at energies above $60 \mathrm{MeV}$ [25]. This makes the high-energy tail of the spectra very important for the determination of the nuclear response [26]. Figure 1 illustrates these distributions and compares them to beta-beam spectra at low gammas. The precise shape of the beta-beam spectra depends on the boost factor $\gamma$ of the primary ion beam and the opening angle of the flux to the target, but is remarkably similar to the supernova neutrino energy distribution. Both classes of distributions are characterized by long tails. The range of the low-energy beta-beam spectra covers the energy region of interest for supernova neutrinos.

We exploit the flexibility offered by beta-beam facilities to construct linear combinations of beta-beam energy distributions,

$$
n_{N \gamma}\left(\varepsilon_{i}\right)=\sum_{i=1}^{N} a_{i} n_{\gamma_{i}}\left(\varepsilon_{i}\right),
$$

where all distributions involved were normalized to 1 :

$$
\int \mathrm{d} \varepsilon_{i} n_{N \gamma}\left(\varepsilon_{i}\right)=1
$$

and

$$
\int \mathrm{d} \varepsilon_{i} n_{\gamma_{i}}\left(\varepsilon_{i}\right)=1, \quad \forall i .
$$

The constructed spectrum that represents the best fit to the original supernova spectrum is then obtained by minimizing the expression

$$
\int_{\varepsilon_{i}} \mathrm{~d} \varepsilon_{i}\left|n_{N \gamma}\left(\varepsilon_{i}\right)-n_{S N}\left(\varepsilon_{i}\right)\right|,
$$

where the similarities between beta-beam and supernova neutrino spectra assure that a good fit is easily obtained. In this way, the values for the expansion parameters $a_{i}$ and the boost factors $\gamma_{i}$ that yield a spectrum that is as close as possible to the original power law distribution are determined. Count rate considerations favor higher gammas, for example between $\gamma$-values between 5 and 15 . Table 1 gives an overview of the count rates that can be expected in a detector $10 \mathrm{~m}$ away from a straight section in a betabeam ring with a length of $90 \mathrm{~m}$, while the target was taken to have an area of $4 \mathrm{~m}^{2}$.
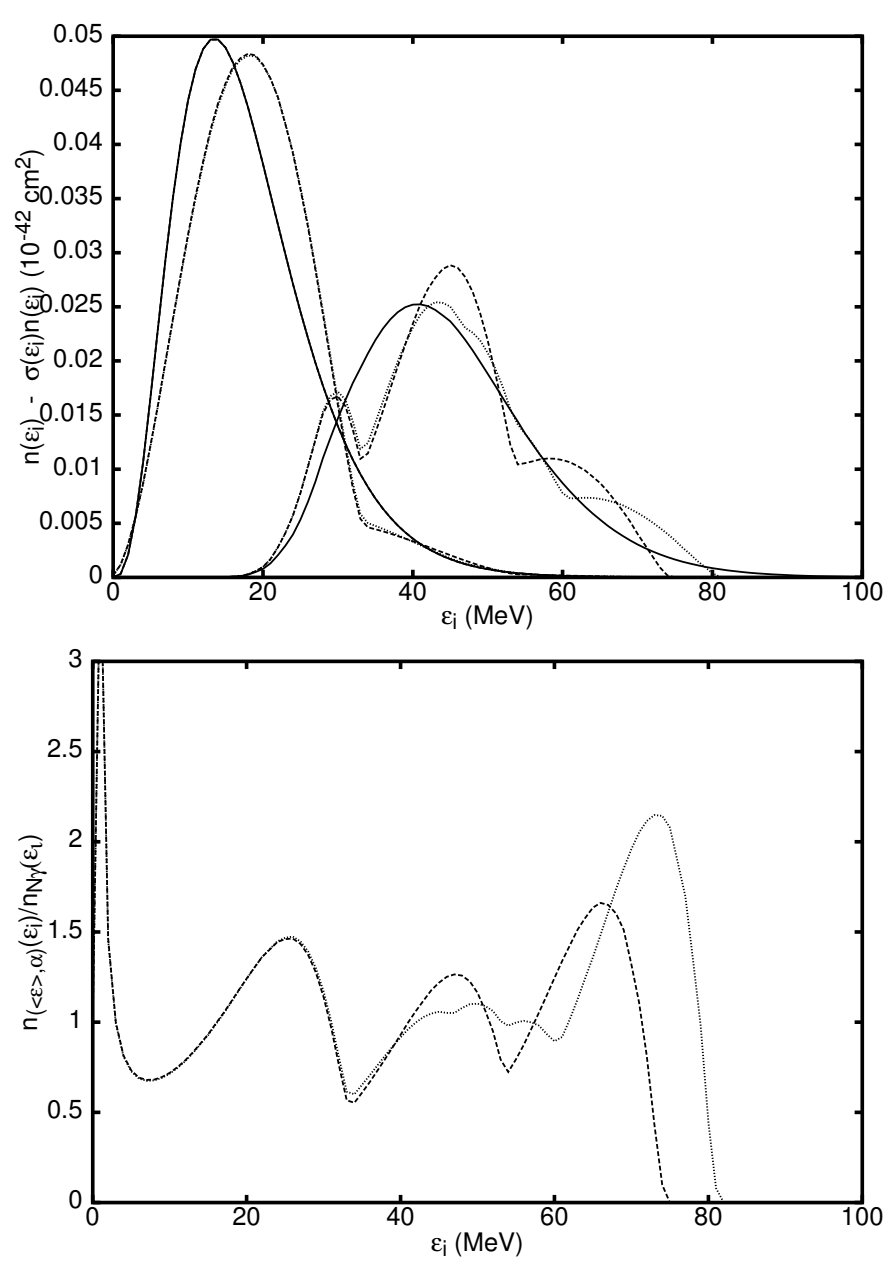

Fig. 2. Comparison between the original and the synthetic spectra for a neutrino energy distribution with average energy $\langle\varepsilon\rangle=18 \mathrm{MeV}$ and $\alpha=3$. In the top panel, the curves to the left represent the spectra, the curves to the right of the plot show total folded cross-sections for neutral-current neutrino scattering off ${ }^{16} \mathrm{O}$. For each set of curves, the full line represents results obtained with the original spectrum, while the other shows the results for calculations with three (dashed) and five (dotted) beta-beam spectra in the fit. The ${ }^{16} \mathrm{O}$ cross-sections were obtained within a continuum random phase approximation calculation $[27,28]$. 

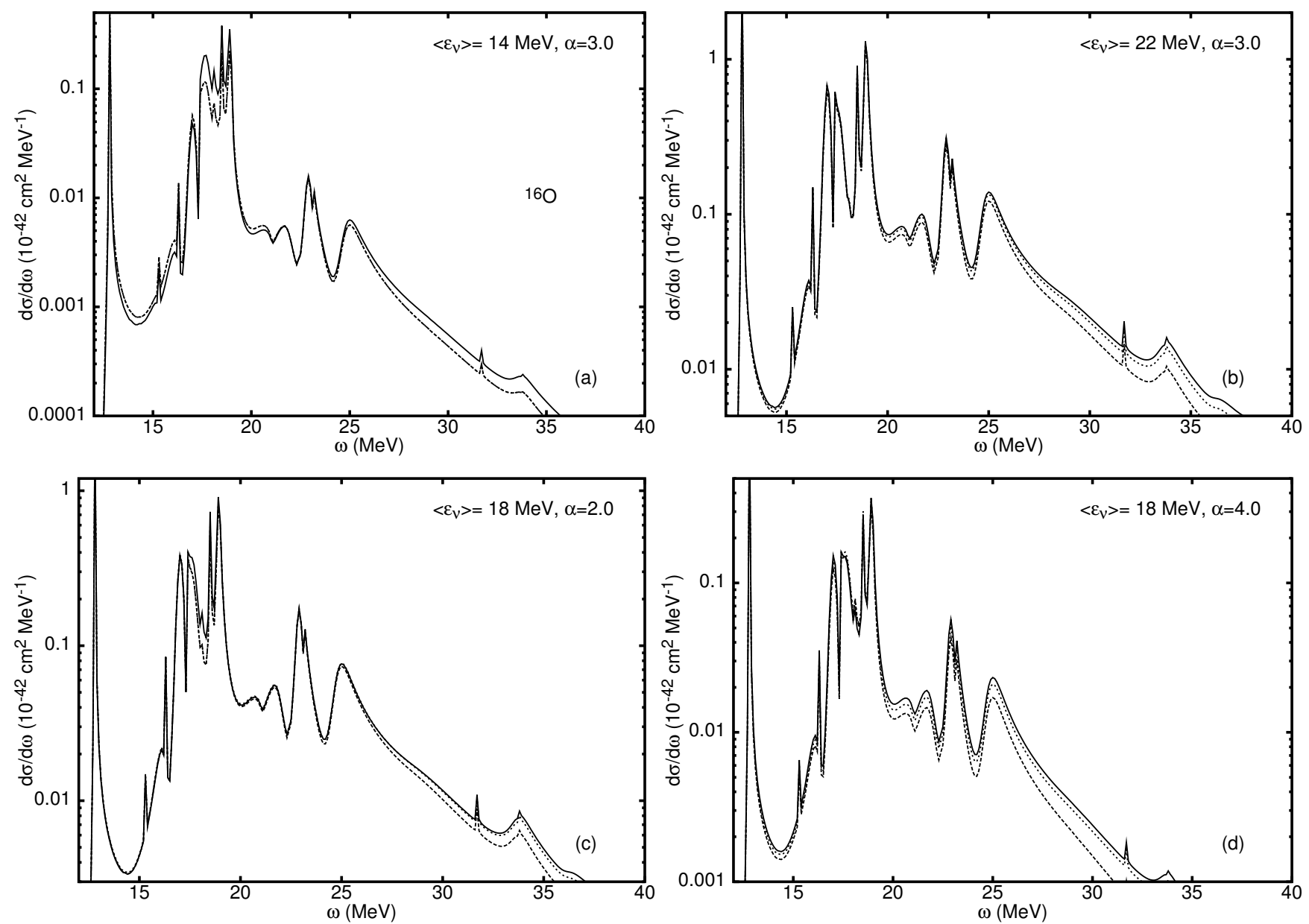

Fig. 3. Comparison between differential cross-sections for neutral-current scattering on ${ }^{16} \mathrm{O}$, folded with a power law supernova neutrino spectrum (full line) and synthetic spectra with 3 (dashed line) and 5 components (dotted line) for different energy distributions: $\langle\varepsilon\rangle=14, \alpha=3$ (a), $\langle\varepsilon\rangle=22, \alpha=3(\mathrm{~b}),\langle\varepsilon\rangle=18, \alpha=2(\mathrm{c})$, and $\langle\varepsilon\rangle=18, \alpha=4$ (d).

Table 2 shows the results of the fitting procedure for a number of relevant energies and widths of the supernova neutrino energy distribution. The parameters are shown for constructions with three and five beta-beam spectra in the fit of eq. (5). Whilst the main contribution stems from spectra at low gamma, contributions from higher gammas are very important for the reproduction of the spectrum's tail. This is illustrated in fig. 2. There we show the original power law spectra, together with the $N=3$ and $N=5$ fits, and the respective folded cross-sections for neutralcurrent scattering off ${ }^{16} \mathrm{O}$ as a function of incident neutrino energy. The figure clearly illustrates that the folded cross-section reaches its main strength for neutrinos in the high-energy part of the distribution. Therefore, it is important that the addition of some spectra at higher $\gamma$ in the fit assures a good agreement between the constructed and the original spectrum in this energy region. The minimization procedure indeed fulfills this demand: whereas the peak of the fitted spectrum is slightly shifted to higher energies, the agreement between the folded cross-sections is rather satisfying. Including five beta-beam spectra in the construction clearly improves the performances of the minimization procedure at higher energies.

\section{Detector response}

Of course the spectrum as such is not an important observable. The information brought along by supernova neutrinos is encoded in the response of the detector to the incoming neutrino flux. This quantity is determined by the folded differential cross-section: the folded cross-section as a function of the excitation energy of the target indicates what the neutrino signal in the detector will look like.

In fig. 3, we show the differential cross-section for neutral-current neutrino scattering on an ${ }^{16} \mathrm{O}$ target for different energy distributions. The agreement between cross-sections folded with the power law supernova neutrino spectrum and those folded with the synthetic spectrum is remarkably good. The procedure is able to reproduce total strength, and the position and width of the resonances very accurately. 

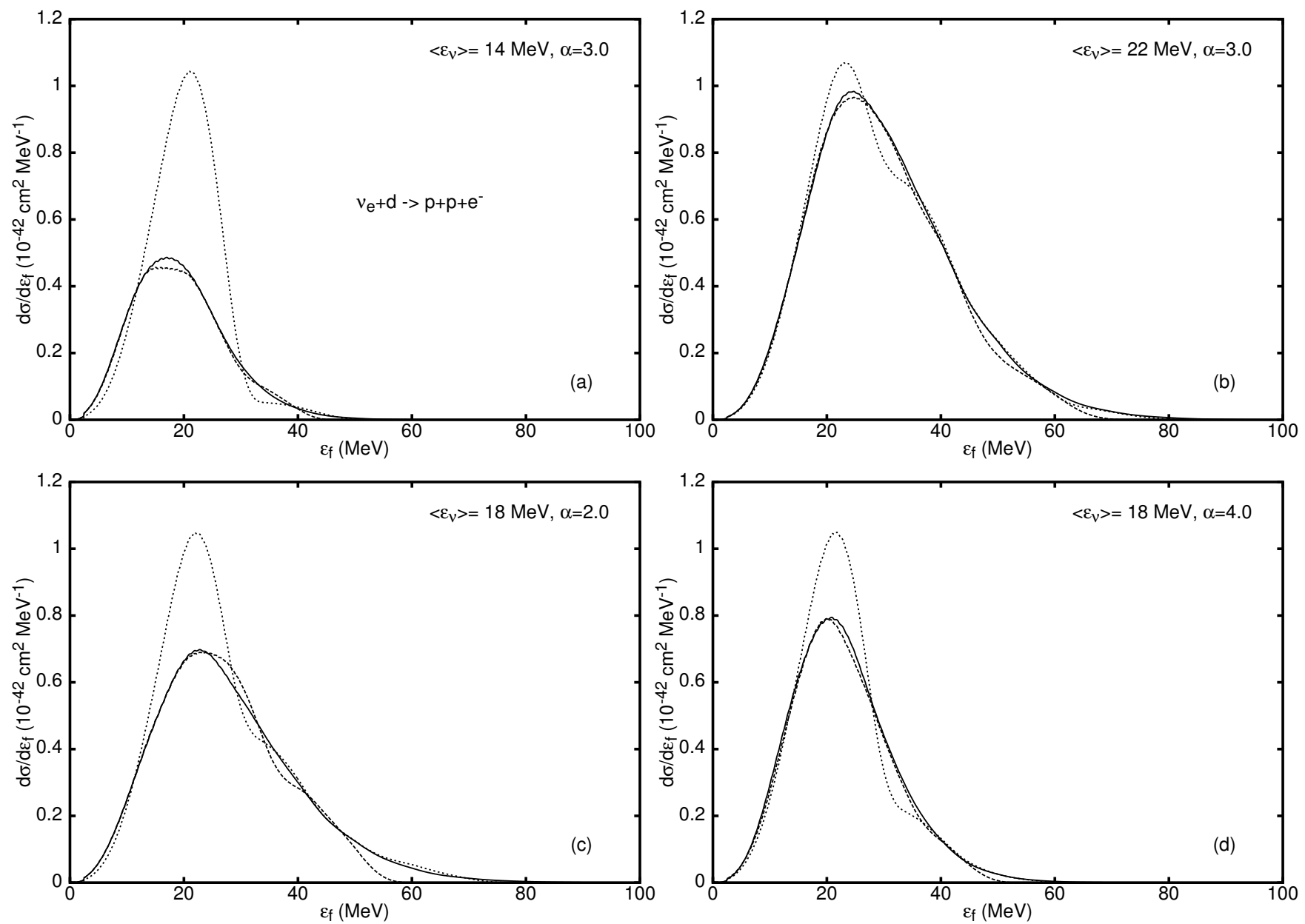

Fig. 4. Comparison between differential cross-sections for the charged-current reaction $\nu_{e}+d \rightarrow p+p+e^{-}$, folded with a power law supernova neutrino spectrum (full line) and synthetic spectra with 5 components, using gamma factors ranging from 5 to 15 (dotted line) and including lower energy beta-beam spectra with $\gamma=2$ to 15 (dashed line), for different energy distributions: $\langle\varepsilon\rangle=14 \mathrm{MeV}, \alpha=3(\mathrm{a}),\langle\varepsilon\rangle=22 \mathrm{MeV}, \alpha=3(\mathrm{~b}),\langle\varepsilon\rangle=18 \mathrm{MeV}, \alpha=2$ (c), and $\langle\varepsilon\rangle=18 \mathrm{MeV}, \alpha=4$ (d). The deuteron cross-sections were obtained from [29].

In principle, the formalism can be applied to any target material. Its efficiency, however, depends on the accuracy of the fitting procedure in the relevant energy range. Figure 4 illustrates differential cross-sections for the reaction $\nu_{e}+d \rightarrow p+p+e^{-}$as a function of the energy of the outgoing electron. For these reactions, thresholds are situated at considerably smaller energy values than for ${ }^{16} \mathrm{O}$ and the cross-section peaks at smaller energies, typically around the maximum of the neutrino distribution. As a consequence, supernova spectra with lower average energies require smaller gamma components in the synthetic spectrum to obtain a good agreement. This is shown in the figure. For fits including beta-beam spectra only down to $\gamma=5$, folded cross-sections at low average energies tend to be overestimated. Including spectra at lower gamma increases the agreement [30]. The introduction of a weight function in the fitting procedure can assure that the fit is optimal in the energy region dominating the nuclear response [30].

\section{Conclusion}

Low-energy beta beams are efficient tools to learn about the response of a neutrino detector to supernova neutrinos. Taking linear combinations of the detector response to beta-beam spectra provides a very accurate picture of the supernova neutrino signal in a terrestrial detector. This technique would work for almost any target nucleus. We have demonstrated theoretically its potential for deuterium and oxygen which are the nuclei relevant for SNO and for SuperKamiokande.

The authors would like to thank C. Volpe, M. Lindroos, and K. Heyde for interesting discussions. N.J. thanks the Fund for Scientific Research Flanders (FWO) for financial support. G.C.M. acknowledges support from the Department of Energy, under contract DE-FG02-02ER41216. 


\section{References}

1. P. Vogel, Prog. Part. Nucl. Phys. 48, 29 (2002); Phys. Rev. C 71, 034604 (2005).

2. J.F. Beacom, R.N. Boyd, A. Mezzacappa, Phys. Rev. D 63, 073011 (2001).

3. J.M. Soares, L. Wolfenstein, Phys. Rev. D 40, 3666 (1989); J.F. Beacom, P. Vogel, Phys. Rev. D 58, 053010 (1998) and references therein.

4. N. Jachowicz, K. Vantournhout, J. Ryckebusch, K. Heyde, Phys. Rev. Lett. 93, 082501 (2004).

5. N. Jachowicz, K. Vantournhout, J. Ryckebusch, K. Heyde, Phys. Rev. C 71, 034604 (2005).

6. G.M. Fuller, W.C. Haxton, G.C. McLaughlin, Phys. Rev. D 59, 085005 (1999).

7. J. Engel, G.C. McLaughlin, C. Volpe, Phys. Rev. D 67, 013005 (2003).

8. V. Barger, P. Huber, D. Marfatia, Phys. Lett. B 617, 167 (2005).

9. The SNO Collaboration (C.J. Clarence), Nucl. Phys. Proc. Suppl. 100, 326 (2001)

10. Y. Oyama et al., Phys. Rev. Lett. 56, 2604 (1987).

11. M. Aglietta, P. Antonioli, G. Bari, C. Castagnoli et al., Nucl. Phys. Proc. Suppl. 138, 115 (2005).

12. M.K. Sharp, J.F. Beacom, J.A. Formaggio, Phys. Rev. D 66, $013012(2002)$.
13. R.N. Boyd, A.St.J. Murphy, Nucl. Phys. A 688, 386c (2001).

14. C.K. Hargrove et al., Astropart. Phys. 5, 183 (1996).

15. L. Oberauer, Mod. Phys. Lett. A 19, 337 (2004).

16. B.E. Bodmann et al., Phys. Lett. B 332, 251 (1994).

17. http://www.phy.ornl.gov/nusns.

18. K. Langanke, G. Martínez-Pinedo, P. von Neumann-Cosel, A. Richter, Phys. Rev. Lett. 93, 202501 (2004).

19. P. Zuchelli, Phys. Lett. B 532, 166 (2002).

20. C. Volpe, J. Phys. G 30, 1 (2004).

21. J. Serreau, C. Volpe, Phys. Rev. C 70, 055502 (2004).

22. G.C. McLaughlin, Phys. Rev. C 70, 045804 (2004).

23. http://beta-beam.web.cern.ch/beta-beam.

24. M. Keil, G.G. Raffelt, H.-T. Janka, Astrophys. J. 590, 971 (2003).

25. N. Jachowicz, K. Vantournhout, J. Ryckebusch, K. Heyde, Nucl. Phys. A 758, 51c (2005).

26. N. Jachowicz, K. Heyde, Phys. Rev. C 68, 055502 (2003).

27. N. Jachowicz, S. Rombouts, K. Heyde, J. Ryckebusch, Phys. Rev. C 59, 3246 (1999).

28. N. Jachowicz, K. Heyde, J. Ryckebusch, Phys. Rev. C 66, 055501 (2002).

29. S. Nakamura et al., Nucl. Phys. A 707, (2002) 561; private communication.

30. N. Jachowicz, G.C. McLaughlin, submitted to Phys. Rev. Lett.. 